\title{
Impact of Hyaluronic Acid-Containing Artificial Tear Products on Reepithelialization in an In Vivo Corneal Wound Model
}

\author{
Eric Carlson,' Winston W.Y. Kao, and Abayomi Ogundele ${ }^{1}$
}

\begin{abstract}
Purpose: To evaluate the effect of 6 commercially available hyaluronic acid (HA)-containing topical artificial tear products on corneal reepithelialization following injury, in an in vivo mouse model.

Methods: Ninety-six C57B1/6 mice (16 per treatment group; male to female ratio, 1:1 per group) were anesthetized. Epithelial debridement was performed on 1 cornea per animal, and the debrided eye was imaged. A $30 \mu \mathrm{L}$ masked test solution containing 1 of 6 artificial tear products was instilled, immediately on debridement, and subsequently, every $2 \mathrm{~h}$, for a total of 4 administrations. At $24 \mathrm{~h}$ post debridement, corneas were stained with fluorescein and imaged to calculate corneal healing rate (number of fluorescein-negative corneas).

Results: All 6 artificial tear products used in this study permitted the initial process of corneal wound healing. However, the corneal reepithelialization rate after $24 \mathrm{~h}$ was higher with Hydroxypropyl guar (HPG)/HA (53.33\%) compared with other HA-containing artificial tear products [HA1 (12.5\%), HA2 (26.67\%), HA3 $(31.25 \%)$, HA4 (6.25\%), and HA5 (43.75\%)]. The average area and percentage area of reepithelialization after $24 \mathrm{~h}$ were also higher with HPG/HA compared with other treatment groups.

Conclusions: Percentage of eyes with complete corneal reepithelialization $24 \mathrm{~h}$ post debridement was highest with HPG/HA compared with other HA-containing artificial tear products tested. The results of this study provide additional evidence on the potential benefits of HPG/HA in the management of dry eye and its role in the rapid restoration of a healthy ocular epithelium. However, further studies are required to confirm the effects on human corneal wounds.
\end{abstract}

Keywords: cornea, corneal epithelium, dry eye, wound healing

\section{Introduction}

T HE HUMAN CORNEA is an avascular, transparent tissue. The corneal epithelium is the outermost layer of the cornea, which is $\sim 50 \mu \mathrm{m}$ in thickness. The tear film, which is $\sim 8 \mu \mathrm{m}$ in thickness, covers the ocular surface. The tear film-corneal epithelial interface provides a proper anterior refractive surface for the eye, and acts as the primary infectious and structural barrier of the eye. ${ }^{1}$

The most superficial layers of the corneal epithelium are flat and polygonal in shape with apical microvilli and microplicae, and are covered by a charged glycocalyx. ${ }^{2}$ The close interaction between the mucinous layer of the tear film and the corneal epithelial cell glycocalyx allows hydrophilic spreading of the tear film with each eyelid blink. The glycocalyx increases the surface area of contact and adherence between the tear film and the epithelial membrane, which is critical for a smooth and clear optical system. Hence, loss of the corneal epithelial glycocalyx due to injury or disease affects the tear film stability and leads to breakdown of the ocular optical system. ${ }^{1}$ Disruption in the tear film stability caused by ocular surface diseases and injuries, such as dry eye, allergies, scarring from glaucoma medications, immunological conditions, chemical/thermal injuries, and refractive surgery, can lead to the corneal epithelial injury. ${ }^{3}$

The corneal epithelium is capable of self-renewal, and the healing of corneal wound is guided by a series of events that include cell death, migration, proliferation, adhesion, differentiation, and extracellular matrix remodelling. ${ }^{4,5}$ Glycosaminoglycans (GAGs), the most abundant heteropolysaccharides in the human eye, are responsible for providing plasticity and structural support needed for successful corneal

\footnotetext{
${ }^{1}$ Novartis Pharmaceuticals Corporation, Fort Worth, Texas.

${ }^{2}$ Department of Ophthalmology, College of Medicine at the University of Cincinnati, Cincinnati, Ohio.
}

(C) Eric Carlson et al. 2018; Published by Mary Ann Liebert, Inc. This article is available under the Creative Commons License CC-BY-NC (http://creativecommons.org/licenses/by-nc/4.0). This license permits non-commercial use, distribution and reproduction in any medium, provided the original work is properly cited. Permission only needs to be obtained for commercial use and can be done via RightsLink. 
Table 1. The Active Ingredient and Preservative in Each of the 6 Artificial Tear Products

Artificial

tear product

Active ingredient

\begin{tabular}{ll}
\hline HA1 & HA $0.1 \%$ and CMC \\
HA2 & HA $0.18 \%$ \\
HA3 & Trehalose $3 \%$ and HA $0.15 \%$ \\
HA4 & HA $0.15 \%$ \\
HA5 & HA $0.1 \%$ provided in the COMOD device \\
HPG/HA & HPG, HA $0.1 \%$, and demulcents
\end{tabular}

CMC, carboxymethylcellulose; HA, hyaluronic acid; HA1 (Optive Fusion ${ }^{\mathrm{TM}}$; Allergan Limited, United Kingdom); HA2 (Vismed ${ }^{\circledR}$; TRB Chemedica International S.A., Switzerland); HA3 (Thealoz Duo ${ }^{\circledR}$; Thea Pharmaceuticals Limited, United Kingdom); HA4 (Hyabak ${ }^{\circledR}$; Thea Pharmaceuticals Limited, United Kingdom); HA5 (Hylo-Comod ${ }^{\circledR}$; URSAPHARM Arzneimittel GmbH, Germany); and HPG/HA (Systane $^{\circledR}$ Hydration; Alcon Research Ltd., Fort Worth Texas); HPG/HA, hydroxypropyl guar/hyaluronic acid-containing dual-polymer lubricant formulation.

function. ${ }^{6}$ Hyaluronic acid (HA), a naturally occurring endogenous GAG, has a key role in each phase of corneal epithelial wound healing, including cell proliferation, antiinflammation, and wound repair. ${ }^{7} \mathrm{HA}$ is viscoelastic and has a high water-retention capacity, and is therefore widely used in artificial tears to treat dry eye disease by improving ocular hydration and reducing surface friction. ${ }^{7} \mathrm{HA}$ also plays an important role in tissue development and wound healing by promoting early inflammation. ${ }^{8}$ Sodium hyaluronate containing artificial tears has been reported to have a beneficial effect on the conjunctival epithelium in patients with dry eye. ${ }^{7}$

Hydroxypropyl guar (HPG) is a viscous mucomimetic polymer, that has also been shown to reduce ocular surface friction. ${ }^{9}$ On exposure to the $\mathrm{pH}$ of tears and ocular surface, HPG forms a soft gel with increased viscosity, and its bioadhesive properties promote the retention of the demulcents (a substance that relieves irritation of the mucous membranes by forming a protective film with its oily or mucilaginous consistency) and protect the ocular surface microenvironment, thus decreasing tear evaporation and increasing tear film stability. ${ }^{9}$ In an in vitro model of corneal epithelium, the dual polymer formulation of HPG/HA demonstrated enhanced ocular surface hydration, surface retention, cell barrier function, and lubricity compared to single-polymer formulations. ${ }^{10}$

In this in vivo preclinical study, we evaluated the effect of 6 marketed HA-containing artificial tear products on reepithelialization of a corneal debridement wound following topical ocular administration, using a mouse model.

\section{Methods}

A total of $96 \mathrm{C} 57 \mathrm{Bl} / 6$ mice (48 male, 48 female, 8-10 weeks old) were used in this study. Sixteen mice (purchased from the Jackson's laboratory, Memphis, TN) were randomized (1:1 ratio of male to female per group) to each of the 6 artificial tear products [Optive Fusion ${ }^{\mathrm{TM}}$, Allergan Limited, United Kingdom (HA1); Vismed ${ }^{\circledR}$, TRB Chemedica International S.A., Switzerland (HA2); Thealoz Duo ${ }^{\circledR}$, Thea Pharmaceuticals Limited, United Kingdom (HA3); Hyabak $^{\circledR}$, Thea Pharmaceuticals Limited, United Kingdom (HA4); Hylo-Comod ${ }^{\circledR}$, URSAPHARM Arzneimittel GmbH, Germany (HA5); and Systane ${ }^{\circledR}$ Hydration, Alcon Research Ltd., Fort Worth Texas, USA (HPG/HA, a dual polymer containing HPG and HA)]. The active ingredient and preservative in each of the 6 artificial tear products are presented in Table 1.
Animals were maintained on a 14-h light (lights were on from 6.00 am to $8.00 \mathrm{pm}$ )/10-h dark cycles at the Laboratory Animal Management System facility (LAMS, Cincinnati, $\mathrm{OH})$. Epithelium debridement was performed by demarcating the cornea using a $2 \mathrm{~mm}$ trephine, ${ }^{11}$ and then utilizing an Algerbrush $^{\circledR}$ II (Accutome, Inc., Malvern, PA) with a $1 \mathrm{~mm}$ burr on 1 cornea per animal (Fig. 1) for $\sim 1$ min (basement membrane was not damaged under stereo microscope examination with fluorescein staining). Each debrided eye was imaged immediately after debridement $(0 \mathrm{~h})$, and then, $30 \mu \mathrm{L}$ of the masked test eye drops containing 1 of the 6 artificial tear products were topically administered to the ocular surface at $0 \mathrm{~h}$, and subsequently, every $2 \mathrm{~h}$, for a total of 4 administrations. The injured corneas were allowed to heal for $24 \mathrm{~h}$. Every animal (4 mice/cage) was subjected to surgery at different time points. The exact time of surgery was recorded and animals were treated with artificial tear products, 1 drop every $2 \mathrm{~h}$ for $6 \mathrm{~h}$ from the time of surgery, and examined exactly $24 \mathrm{~h}$ later at the end of debridement (within $1 \mathrm{~min}$ from beginning to completion). All surgeries were done in the morning from 9:00 am for first mouse to 12:00 pm for the last mouse, treated accordingly, and returned to housing $24 \mathrm{~h}$ later. Twenty-four hours after debridement, corneas were stained with fluorescein, imaged [1 image/animal at each time point $(0 \mathrm{~h}$, at the completion of surgery and $24 \mathrm{~h}$ after surgery)], and analyzed using Adobe Photoshop CS6 (Adobe Systems Incorporated, San Jose, CA) to calculate the total fluorescein positive area (in pixels). The investigator inducing the ocular wound, administering test articles, imaging, and analyzing the data was blinded. Test articles were supplied with only alphanumeric labels, which was not indicative of the artificial tear product.

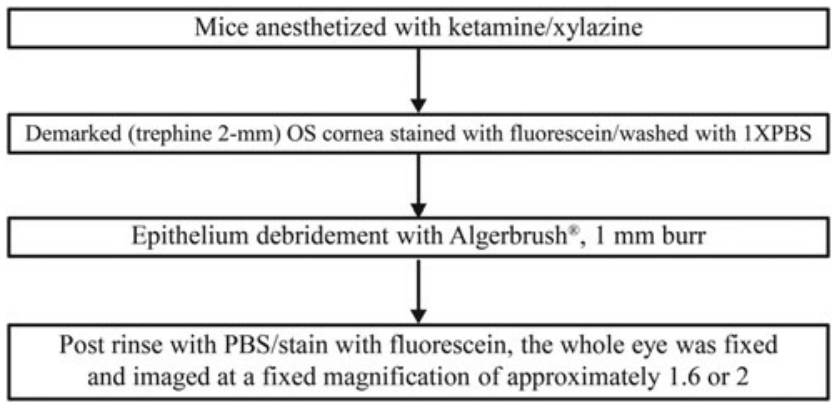

FIG. 1. Epithelium debridement procedure. OS, left eye; PBS, phosphate-buffered saline. 
a HA1 treatment group
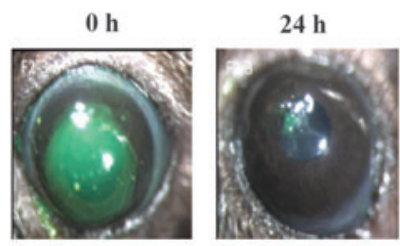

d HA4 treatment group
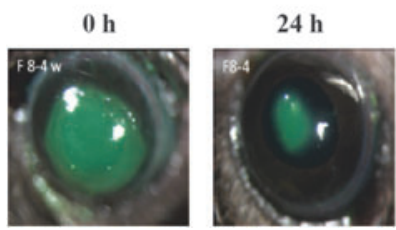

b

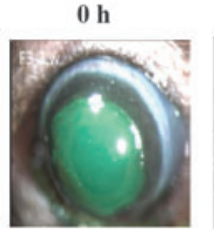

e

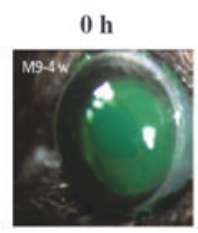

HA2 treatment group

$24 \mathrm{~h}$

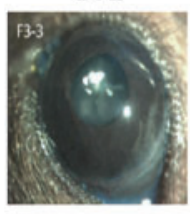

ment group

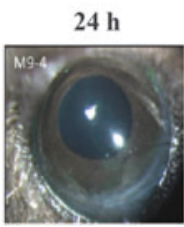

C HA3 treatment group

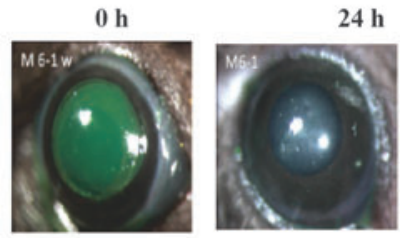

f HPG/HA treatment group
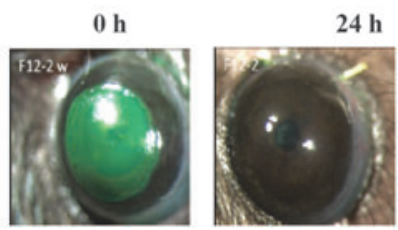

FIG. 2. Representative images showing corneal epithelium before and $24 \mathrm{~h}$ post debridement in each treatment group (a-f) (green staining on images indicate wound area). HA, hyaluronic acid; HA1, Optive Fusion ${ }^{\mathrm{TM}}$, Allergan Limited, United Kingdom; HA2, Vismed ${ }^{\circledR}$, TRB Chemedica International S.A., Switzerland; c) HA3, Thealoz Duo ${ }^{\circledR}$, Thea Pharmaceuticals Limited, United Kingdom; HA4, Hyabak ${ }^{\circledR}$, Thea Pharmaceuticals Limited, United Kingdom; HA5, Hylo-Comod ${ }^{\circledR}$, URSAPHARM Arzneimittel GmbH, Germany; and HPG/HA, Systane ${ }^{\circledR}$ Hydration, Alcon Research Ltd., Fort Worth Texas, USA; HPG/HA, hydroxypropyl guar/hyaluronic acid-containing dual-polymer lubricant formulation.

Animal care and use conformed to the Association for Research in Vision and Ophthalmology (ARVO) Statement for the Use of Animals in Ophthalmic and Vision Research. ${ }^{12}$ All animal protocols were approved by the Institutional Animal Care and Use Committee of the University of Cincinnati and adhered to the guidelines established in the Guide for the Care and Use of Laboratory Animals.

The key endpoints of the study were to evaluate the number of reepithelialized corneas (fluorescein negative) in each treatment group $24 \mathrm{~h}$ after debridement, and to evaluate the rate of corneal reepithelialization in each treatment group $24 \mathrm{~h}$ after debridement.

\section{Statistical analysis}

A statistical difference between the HPG/HA eye drop and the other HA-containing artificial tear products for the mean difference in average reepithelialized area was evaluated using 1-way analysis of variance with Dunnett's multiple comparison test. $P$ value of less than 0.05 was considered statistically significant. Descriptive statistics was

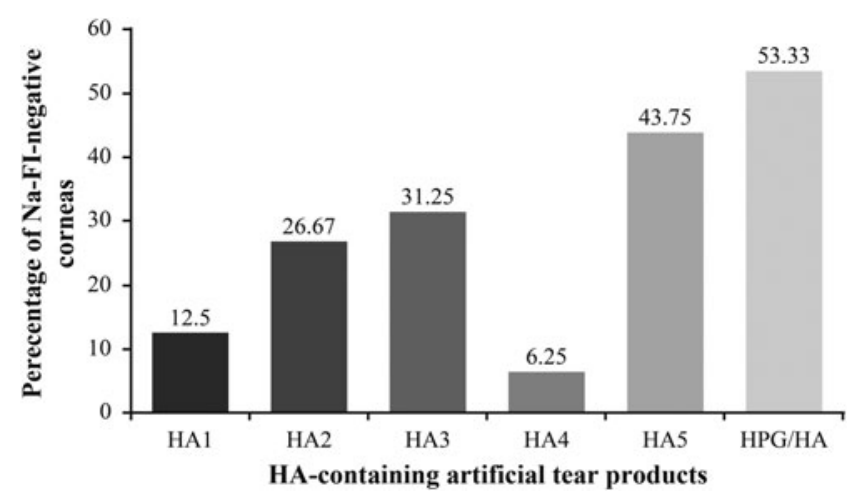

FIG. 3. The number of reepithelialized corneas in each treatment group $24 \mathrm{~h}$ post debridement. FI, fluorescein; HA, hyaluronic acid; HPG, hydroxypropyl-guar; Na, sodium. used to summarize the percentage of reepithelialized corneas (fluorescein negative) in each treatment group.

\section{Results}

All 6 artificial tear products used in this study permitted the initial process of corneal wound healing. Figure 2 shows the corneal fluorescein-stained images of corneas, representative of each treatment group, immediately after debridement $(0 \mathrm{~h})$ and $24 \mathrm{~h}$ post debridement (after instillation with the HA-containing artificial tear products).

The percentage of corneas that were fluorescein negative, $24 \mathrm{~h}$ after debridement, was highest in the HPG/HA treatment group compared with other HA-containing artificial tear products (Fig. 3). Similarly, $24 \mathrm{~h}$ after corneal epithelial debridement, the average area of reepithelialization (pixels) was significantly higher in the HPG/HA versus each of the other HA-containing treatment groups (Fig. 4), and the percentage area of reepithelialization was highest in the HPG-/HA-treated corneas versus those treated with the other HA-containing artificial tear products.

In each of the treatment groups, there was no difference between male and female mice in the rate of reepithelialization.

\section{Discussion}

In this preclinical study, all 6 HA-containing artificial tear products permitted the initial process of corneal wound healing, although the rate of reepithelialization was variable across the treatment groups. Overall, these results support the beneficial role of HA-containing artificial tears in corneal wound healing. The corneal reepithelialization rate was faster in mouse corneas instilled with the dual polymer HPG/HA solution than in those instilled with other HAcontaining artificial tear products tested. Both male and female mice were included to see if there was any difference in the rate of reepithelialization process between the genders. Our results showed that there were no differences 


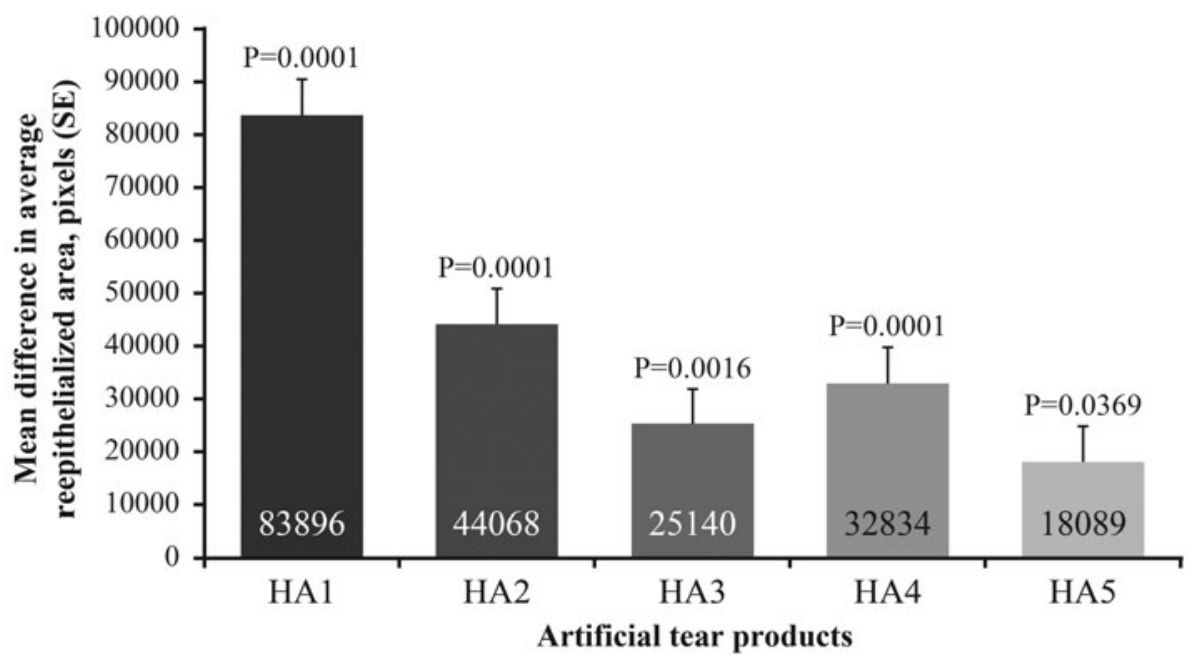

\begin{tabular}{lccc}
\hline Treatment group & $\begin{array}{c}\text { Average area debrided } \\
\text { 0 hours (pixels) before treatment }\end{array}$ & $\begin{array}{c}\text { Average area reepithelialized } \\
\text { 24 hours (pixels) after treatment }\end{array}$ & $\begin{array}{c}\text { Average \% area } \\
\text { reepithelialized }\end{array}$ \\
\hline HA1 & 98875.18 & 90770.75 & 91.89 \\
HA2 & 135794.75 & 130598.73 & 96.64 \\
HA3 & 153445.75 & 149526.56 & 97.38 \\
HA4 & 154824.75 & 141832.53 & 91.61 \\
HA5 & 161925.31 & 156577.62 & 96.75 \\
HPG/HA & 176067.43 & 174666.8 & 98.59 \\
\hline
\end{tabular}

FIG. 4. Difference in the average area of reepithelialization $24 \mathrm{~h}$ post debridement across treatment groups when compared to HPG/HA. The mean difference in average reepithelialized area was calculated and presented by the number of pixels. The statistical difference in the area of reepithelialization between $\mathrm{HPG} / \mathrm{HA}$ group and HAcontaining products was evaluated using a 1-way analysis of variance model. A $P$ value of less than 0.05 was considered statistically significant. The table provides the average area and percentage area of reepithelialization in each treatment group $24 \mathrm{~h}$ after debridement. HA, hyaluronic acid; HPG, hydroxypropyl guar; SE, standard error.

based on gender. The average $\%$ area reepithelialized and the number of corneas showing complete reepithelialization within $24 \mathrm{~h}$ after corneal debridement was highest in the HPG/HA treatment group (98.6\% and $>50 \%$, respectively) compared with other HA-containing treatment groups.

Previous studies have shown that the use of HAcontaining eye drops in patients with dry eyes have provided improved vitality of corneal epithelial cells and increased barrier function. ${ }^{13,14}$ However, depending on the source of HA (plant or animal), and its mucoadhesive property and viscosity, the time period of contact with cornea and bioavailability may vary even at the same HA concentration. ${ }^{15,16}$ Based on previous in vivo and in vitro study results, HA is known to promote corneal wound healing by triggering corneal epithelial cell proliferation and migration. ${ }^{17-22}$ This effect is seen to be concentration-dependent and can be considered a pure pharmacological effect of HA. ${ }^{21,22}$

Similarly, in vivo studies have shown that HPG demonstrates greater corneal epithelial cell viability, better cell barrier function, and increased tear film stability, thus, providing long-lasting ocular surface hydration. ${ }^{23,24}$ HPG is known to enhance the retention of demulcents and HA on the corneal surface after combining with the borate ions (by forming a crosslink to provide a gel-like matrix) when used in the lubricant eye drops. ${ }^{9,25}$ Hence, the use of HPG in artificial tear products containing HA leads to longer HA retention on ocular surface and thus provides better corneal wound healing. $^{22}$

A healthy ocular surface is essential for proper vision and dry eye conditions compromises the patients' quality of life. In severe cases, the inflammation associated with dry eye syndrome could potentially result in irreparable damage to the corneal surface. ${ }^{19}$ Artificial tears such as those containing HA, HPG, or HA/HPG combination are the mainstay of therapy for reducing signs and symptoms associated with dry eye.
Of these, the use of HPG/HA combination may be expected to provide synergistic benefits in reducing ocular surface damage in dry eye disease by reducing surface friction, as well as aiding in the regeneration/repair of damaged epithelial layer. A previous in vitro study demonstrated effective hydration and lubrication and long-lasting effect of HPG/HA combination (along with propylene glycol and polyethylene glycol) compared with other HA- or HPGalone formulations. ${ }^{10}$ The results of this in vivo study demonstrated improved corneal reepithelialization rates with HPG/ HA over other HA-containing single-polymer artificial tear products, further supporting the potential benefits of HPG/ HA dual polymer in the treatment of dry eye. ${ }^{10}$ HPG-/ HA-containing formulations provide prolonged hydration immediately after treatment and lubrication (after the formulation was rinsed away), with reduced ocular surface damage associated with tissue desiccation and increased friction. This dual-polymer formulation has also demonstrated greater cell-cell barrier protection from surfactant insult, providing effective hydration and lubrication, and may protect the ocular surface from damage associated with dry eye. ${ }^{10}$ The limitations of this study are the use of an in vivo preclinical model, which limits extrapolation of study results to clinical application, the surgically/ mechanically induced corneal epithelial injuries in a controlled environment (without damaging the basement membrane or corneal stroma), and discontinuation of the study at $24 \mathrm{~h}$. The study also did not have a control group.

\section{Conclusions}

This study provides evidence on the synergistic benefits of the HPG/HA dual polymer on improving corneal reepithelialization rates over other single HA or dual-polymer HA-containing artificial tear products tested. The study results provide additional evidence on the benefits of HPG/HA 
in the management of dry eye, as it may also play a role in the rapid restoration of a healthy ocular epithelium. However, further studies in humans are required to confirm the benefits of HPG/HA on human corneal wounds.

\section{Acknowledgments}

Novartis Pharmaceuticals Corporation (Fort Worth, TX) sponsored this study. Medical writing and editorial assistance was provided by Rhutika Desai and Lakshmi Venkatraman (Scientific Services Practice-Product Lifecycle Services, Novartis Healthcare Pvt. Ltd., Hyderabad, India) toward the development of this article.

\section{Author Disclosure Statement}

Alcon Research Ltd, is a Novartis Company. A.O. is an employee of Novartis Pharmaceuticals Corporation, Fort Worth, TX, and E.C. was an employee of Novartis Pharmaceuticals Corporation, Fort Worth, TX, at the time of the study. W.W.Y.K. received grants from Research to Prevent Blindness and Ohio Lions Eye Research Foundation (NIH/ NEI grants 011845).

\section{References}

1. DelMonte, D.W., and Kim, T.J. Anatomy and physiology of the cornea. Cataract Refract. Surg. 37:588-598, 2011.

2. Eghrari, A.O., Riazuddin, A., and Gottsch J.D. Overview of the cornea: structure, function, and development. Prog. Mol. Biol. Transl. Sci. 134:7-23, 2015.

3. Optometric Clinical Practice Guideline. American Optometric Association. www.aoa.org/documents/optometrists/ CPG-10.pdf Accessed March 8, 2017.

4. Facts About the Cornea and Corneal Disease. National Eye Institute, National Institutes of Health. https://nei.nih.gov/ health/cornealdisease Accessed December 24, 2016.

5. Ljubimov, A.V., and Saghizadeh, M. Progress in corneal wound healing. Prog. Retin. Eye Res. 49:17-45, 2015.

6. Pacella, E., Pascella, F., De Paolis, G., et al. Glycosaminoglycans in the human cornea: age-related changes. Ophthalmol. Eye Dis. 7:1-5, 2015.

7. Saranraj, P., and Naidu, M.A. Hyaluronic acid production and its applications-a review. Int. J. Pharm. Biol. Sci. Arch. 4:853-859, 2013.

8. Aragona, P., Papa, V., Micali, A., Santocono, M., and Milazzo, G. Long term treatment with sodium hyaluronatecontaining artificial tears reduces ocular surface damage in patients with dry eye. Br. J. Ophthalmol. 86:181-184, 2002.

9. Christensen, M.T., Cohen, S., Rinehart, J., et al. Clinical evaluation of an HP-guar gellable lubricant eye drop for the relief of dryness of the eye. Clin. Ther. 32:44-52, 2010.

10. Rangarajan, R., Kraybill, B., Ogundele, A., and Ketelson, $\mathrm{H}$. Effects of a hyaluronic acid/hydroxypropyl guar artificial tear solution on protection, recovery, and lubricity in models of corneal epithelium. J. Ocul. Pharmacol. Ther. 31:491-497, 2015.

11. Carlson, E.C., Wang, I.J., Liu, C.Y., Brannan, P., Kao, C.W., and Kao, W.W. Altered KSPG expression by keratocytes following corneal injury. Mol. Vis. 21:615-623, 2003.
12. ARVO Statement for the Use of Animals in Ophthalmic and Vision Research. Association for Research in Vision and Ophthalmology. www.arvo.org/about_arvo/policies/ statement_for_the_use_of_animals_in_ophthalmic_and_ visual_research Accessed May 25, 2017.

13. Troiano, P., and Monaco, G. Effect of hypotonic $0.4 \%$ hyaluronic acid drops in dry eye patients: a cross-over study. Cornea. 27:1126-1130, 2008.

14. Yokoi, N., Komuro, A., Nishida, K., and Kinoshita, S. Effectiveness of hyaluronan on corneal epithelial barrier function in dry eye. Br. J. Ophthalmol. 81:533-536, 1997.

15. Salzillo, R., Schiraldi, C., Corsuto, L., et al. Optimization of hyaluronan-based eye drop formulations. Carbohydr. Polym. 153:275-283, 2016.

16. Baranowski, P., Karolewicz, B., Gajda, M., and Pluta, J. Ophthalmic drug dosage forms: characterisation and research methods. ScientificWorldJournal. 18:861904, 2014.

17. Nishida, T., Nakamura, M., Mishima, H., and Otori, T. Hyaluronan stimulates corneal epithelial migration. Exp. Eye Res. 53:753-758, 1991.

18. Inoue, M., and Katakami, C. The effect of hyaluronic acid on corneal epithelial cell proliferation. Invest. Ophthalmol. Vis. Sci. 34:2313-2315, 1993.

19. Sugiyama, T., Miyauchi, S., Machida, A., Miyazaki, K., et al. The effect of sodium hyaluronate on the migration of rabbit corneal epithelium. II. The effect of topical administration. J. Ocul. Pharmacol. 7:53-64, 1991.

20. Nakamura, M., Hikida, M., and Nakano, T. Concentration and molecular weight dependency of rabbit corneal epithelial wound healing of hyaluronan. Curr. Eye Res. 11: 981-986, 1992.

21. Camillieri, G., Bucolo, C., Rossi, S., and Drago, F. Hyaluronan-induced stimulation of corneal wound healing is a pure pharmacological effect. J. Ocul. Pharmacol. Ther. 20:548-553, 2004.

22. Daull, P., Feraille, L., Elena, PP., and Garrigue, JS. Comparison of the anti-inflammatory effects of artificial tears in a rat model of corneal scraping. J. Ocul. Pharmacol. Ther. 32:109-118, 2016.

23. Ubels, J.L., Clousing, D., Haitsma, T.V., et al. Pre-clinical investigation of the efficacy of an artificial tear solution containing hydroxypropylguar as a gelling agent. Curr. Eye Res. 28:437-444, 2004.

24. Ousler, G.W., Michaelson, C., and Christensen, M.T. An evaluation of tear film breakup time extension and ocular protection index scores among three marketed lubricant eye drops. Cornea. 26:949-952, 2007.

25. Kesavan, S., and Prud'homme, RK. Rheology of guar and HPG cross-linked by borate. Macromolecules. 25:20262032, 1992.

Received: July 11, 2017 Accepted: November 20, 2017

Address correspondence to: Dr. Abayomi Ogundele

Novartis Pharmaceutical Corporation (Alcon Campus) 6201 South Freeway

Fort Worth, TX 76134-2099

E-mail: abayomi.ogundele@novartis.com 